\title{
APPS 2020: Second International Workshop on Adaptive and Personalized Privacy and Security
}

\author{
Marios Belk \\ Cognitive UX GmbH, Heidelberg, Germany \\ University of Cyprus, Nicosia, Cyprus \\ belk@cognitiveux.de
}

\author{
Elias Athanasopoulos \\ University of Cyprus, Nicosia, Cyprus \\ athanasopoulos.elias@cs.ucy.ac.cy
}

\author{
Christos Fidas \\ University of Patras \\ Rio, Patras, Greece \\ fidas@upatras.gr
}

\author{
Juliana Bowles \\ University of St. Andrews \\ Scotland, UK \\ jkfb@st-andrews.ac.uk \\ Andreas Pitsillides \\ University of Cyprus, Nicosia, Cyprus \\ andreas.pitsillides@cs.ucy.ac.cy
}

\begin{abstract}
The Second International Workshop on Adaptive and Personalized Privacy and Security (APPS 2020) aims to bring together researchers and practitioners working on diverse topics related to understanding and improving the usability of privacy and security software and systems, by applying user modeling, adaptation and personalization principles. Our special focus in 2020 is on healthcare systems, more specifically on ensuring security and privacy of medical data in smart patient-centric healthcare systems. The second edition of the workshop includes interdisciplinary contributions from Austria, Canada, China, Cyprus, Denmark, Germany, Greece, Israel, the Netherlands, Turkey and the UK that introduce new and disruptive ideas, suggest novel solutions, and present research results about various aspects (theory, applications, tools) for bringing user modeling, adaptation and personalization principles into privacy and systems security. This summary gives a brief overview of APPS 2020, held online in conjunction with the 28th ACM Conference on User Modeling, Adaptation and Personalization (ACM UMAP 2020).
\end{abstract}

\section{CCS CONCEPTS}

- Human-centered computing • Security and privacy.

\section{KEYWORDS}

Security; Privacy; Usability; Trust; User-centric Systems; Patientcentric Healthcare Systems.

\section{ACM Reference Format:}

Marios Belk, Christos Fidas, Juliana Bowles, Elias Athanasopoulos, Andreas Pitsillides. 2020. APPS 2020: Second International Workshop on Adaptive and Personalized Privacy and Security. In Proceedings of the 28th ACM Conference on User Modeling, Adaptation and Personalization (UMAP '20), fuly 12-18, 2020, Genoa, Italy. ACM, New York, NY, USA, 2 pages. https://doi.org/10.1145/3340631.3398674

\section{INTRODUCTION}

Millions of users across different continents and countries are daily engaged with privacy and security tasks which are indispensable in modern information systems and services. Such tasks are commonly related to user authentication, human interaction proofs (e.g., captcha), privacy and security pop-up dialogs, setting privacy and security features within online user profiles, etc. Recent privacy and security incidents of famous online services have once more underpinned the necessity towards further investigating and improving current approaches and practices related to the design of efficient and effective privacy and security. In order to achieve this objective, one possible direction is related to providing adaptive and personalized features to privacy- and security-related user tasks, given the diversity of the user characteristics (like cultural, cognitive, age, habits), the technology (like standalone, mobile, mixed-virtual-augmented reality, wearables) and interaction contexts of use (like being on the move, social settings, spatial limitations). Hence, adaptive and personalized privacy and security implies the ability of an interactive system or service to support its end-users, who are engaged in privacy- and/or security-related tasks, based on user models which describe in a holistic way what constitutes the user's physical, technological and interaction context in which computation takes place.

APPS 2020 aims to bring together researchers and practitioners working on diverse topics related to understanding and improving the usability of privacy and security software and systems, by applying user modeling, adaptation and personalization principles. Our special focus in 2020 is on healthcare systems, more specifically on ensuring security and 
privacy of medical data in smart patient-centric healthcare systems. The workshop addresses the following objectives:

- increase our understanding and knowledge on supporting usable privacy and security interaction design through novel user modeling mechanisms and adaptive user interfaces;

- discuss methods and techniques for understanding user attitudes and perceptions towards privacy and security issues in various application areas;

- identify human-centered models for the design, development and evaluation of adaptive and personalized privacy and security systems;

- discuss methods for evaluating the impact and added value of adaptation and personalization in privacy and security systems.

\section{Workshop Topics and Themes}

The workshop focuses on the following topics and themes:

- Privacy and security in smart patient-centric healthcare systems;

- Adaptation and personalization approaches in usable privacy and security;

- Effects of human factors (e.g., cognition, personality, etc.) in privacy and security systems;

- Novel user interaction concepts and user interfaces for achieving usable security;

- Cultural diversity in usable privacy and security;

- Context-aware privacy and security;

- Adaptive usable security in various domains such as healthcare, Internet of Things, automotive;

- Trust perceived in patient-centric healthcare systems;

- Perceived security and usability in patient-centric healthcare systems;

- Adaptive user authentication policies;

- Novel approaches for the design and evaluation of usable security systems;

- Lessons learned from the deployment and use of usable privacy and security features;

- Ethical considerations in adaptive and personalized privacy and security.

\section{Workshop Program}

The workshop is held as an online virtual event this year. The program features a short introduction provided by the workshop organizers, and invited talks from researchers related to the area of APPS. The main workshop includes oral presentations by authors of accepted full papers and short contributions, followed by questions and discussion on each paper. This year's edition consists of two tracks: (i) main track on adaptive and personalized privacy and security; and (ii) focused track on patient-centric healthcare systems. The main program has been shaped as follows:

- Invited talks. (i) Esma Aïmeur (University of Montreal, Canada): Towards a Personalized User-Centric Approach to
Raise Awareness of Privacy and Cybersecurity; (ii) Michael Vinov (IBM Haifa Research Lab, Israel): Rule-based Data Fabrication in Patient-centric Healthcare Systems; and (iii) Wanting Huang et al. (Accenture, Netherlands): Blockchain-based Solution for the Serums Project to Securely Share Patient Data Across Europe.

- Full papers. (i) Eelco Herder and Olaf van Maaren: Privacy Dashboards: The Impact of the Type of Personal Data and User Control on Trust and Perceived Risk; (ii) Anna Maria Pietron and Ting Han: A Case Study of Graphical Passwords in a Chinese University; (iii) Sara Abri et al.: Group-based Personalization Using Topical User Profile; and (iv) Mohit Kumar et al.: Differentially Private Learning of Distributed Deep Models.

- Short papers. (i) Tiberiu-Ioan Szatmari et al.: Modeling Audiological Preferences using Federated Learning; (ii) Juliana Bowles et al.: Interacting with Next-Generation Smart Patient-Centric Healthcare Systems; and (iii) Argyris Constantinides et al.: Design and Development of a Patient-centric User Authentication System.

\section{Workshop Organization}

The workshop consists of the following chairs and members of the international program committee:

\subsection{Workshop Chairs}

Marios Belk (Cognitive UX GmbH, Germany \& University of Cyprus, Cyprus); Christos Fidas (University of Patras, Greece); Juliana Bowles (University of St. Andrews, UK); Elias Athanasopoulos (University of Cyprus, Cyprus); Andreas Pitsillides (University of Cyprus, Cyprus).

\subsection{International Program Committee}

We appreciate the reviewers' efforts and would like to thank the members of the international program committee for their valuable support.

Nikolaos Avouris (University of Patras, Greece); Argyris Constantinides (University of Cyprus, Cyprus); Marios Constantinides (Nokia Bell Labs, UK); Andreas Costi (Vrije Universiteit Amsterdam, Netherlands); Bruce Ferwerda (Jönköping University, Sweden); Thomas Given-Wilson (Université Catholique de Louvain, Belgium); George Hadjidemetriou (University of Twente, Netherlands); Heinrich Hussmann (Ludwig Maximilian University of Munich, Germany); Marios Isaakidis (University College London, UK); Georgia Kapitsaki (University of Cyprus, Cyprus); Axel Legay (Université Catholique de Louvain, Belgium); Ben Steichen (California State Polytechnic University, USA).

\section{ACKNOWLEDGMENTS}

The work has been partially supported by the EU Horizon 2020 Grant 826278 "Securing Medical Data in Smart Patient-Centric Healthcare Systems” (Serums). 